\title{
Research Paper:TransdiagnosticProcessesin Generalized Anxiety Disorder and Obsessive-Compulsive Disorder: Worry, Cognitive Avoidance, Intolerance of Uncertainty, and Metacognitive Beliefs
}

\author{
Mohammad Ali Besharat ${ }^{1}$ (D), Mohammad Atari (D), Roghyeh Sadat Mirjalili (D)
}

1. Department of Psychology, Faculty of Psychology and Education, University of Tehran, Tehran, Iran.

\begin{tabular}{|l|l|l}
\hline $\begin{array}{c}\text { Use yur devic to scan } \\
\text { and read thearticle online }\end{array}$ & $\begin{array}{l}\text { Crtation: Besharat, M. A., Atari, M., \& Mirjalili, R. S. (2019). Transdiagnostic Processes in Generalized Anxiety Disorder and } \\
\text { Obsessive-Compulsive Disorder: Worry, Cognitive Avoidance, Intolerance of Uncertainty, and Metacognitive Beliefs. Journal of } \\
\text { Practice in Clinical Psychology, 7(4), 281-290. http://dx.doi.org/10.32598/jpcp.7.4.281 }\end{array}$ \\
doi'http://dx.doi.org/10.32598/jpcp.7.4.281
\end{tabular}

(c) (1) (8)

Article info:

Received: 21 Mar 2019

Accepted: 18 Aug 2019

Available Online: 01 Oct 2019

Keywords:

Cognition, Transdiagnosis, Generalized anxiety disorder, Obsessive-compulsive disorder

\section{ABSTRACT}

Objective: The present study aimed at examining worry, cognitive avoidance, intolerance of uncertainty, and metacognitive beliefs, as transdiagnostic factors, in clinical samples with Generalized Anxiety Disorder (GAD) and Obsessive-Compulsive Disorder (OCD).

Methods: In total, 200 patients (66 with GAD, 74 with OCD, and 60 with comorbid GAD and $\mathrm{OCD}$ ) were selected to participate in this study using the purposeful sampling method. The participants completed the Penn State worry questionnaire, cognitive avoidance questionnaire, intolerance of uncertainty scale, and metacognitive beliefs questionnaire.

Results: The findings showed no significant difference between patients with GAD and OCD in total scores of these constructs; however, the patients with comorbidity of GAD and OCD showed significantly higher scores in these measures $(\mathrm{P}<0.01)$ compared with the patients with GAD or OCD.

Conclusion: These cognitive factors are possible transdiagnostic factors shared between GAD and OCD. Moreover, they considerably increase when GAD and OCD co-occur.

\section{* Corresponding Author:}

Mohammad Ali Besharat, PhD

Address: Department of Psychology, Faculty of Psychology and Education, University of Tehran, Tehran, Iran

Tel: +98 (912) 2207450

E-mail: besharat@ut.ac.ir 


\section{Highlights}

- Four transdiagnostic cognitive factors including worry, cognitive avoidance, intolerance of uncertainty, and metacognitive beliefs were investigated in GAD and/or OCD patients.

- Patients with both GAD and OCD showed significantly higher scores in all transdiagnostic factors compared to the patients with GAD or OCD only.

- Transdiagnostic cognitive factors can be used in transdignostic approaches to the diagnosis and treatment of psychological disorders.

\section{Plain Language Summary}

Mental disorders sometimes include several symptoms which can be caused by different clinical conditions. One of these symptoms is anxiety which can cause generalized anxiety disorder (GAD) and obsessive-compulsive disorder (OCD). These two disorders are common in worry, cognitive avoidance, intolerance of uncertainty and metacognitive beliefs. These components are known as transdiagnostic factors. The present study examined these transdiagnostic factors in a sample of patients with GAD and OCD. Findings showed that when GAD and OCD are present separately, their symptoms seem similar in terms of transdiagnostic cognitive factors; however, when they both are present as comorbid disorders, these factors seems more prevalent and severe. It can be concluded that transdiagnostic factors have a potential for developing comorbidity which requires more attention at prevention phase in clinical settings.

\section{Introduction}

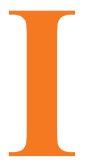

$\mathrm{n}$ the past two decades, psychologists have examined different constructs within a certain psychological disorder, assuming that each disorder has its own distinctive cognitive profile (Brown, Campbell, Lehman, Grisham, \& Mancill, 2001). Yet, considering the high comorbidity in psychological disorders, recent studies have investigated responsible factors for comorbidities, as well as common cognitive factors introduced as transdiagnostic processes (Gillan, Fineberg, \& Robbins, 2017; Harvey, Watkins, Mansell, \& Shafran, 2004; Mansell, Harvey, Watkins, \& Shafran, 2008; McLaughlin \& Nolen-Hoeksema, 2011; Torres, et al., 2016). Anxiety disorders are among the most prevalent psychological disorders and are highly comorbid. In a large-scale study, $43 \%$ of patients had more than one anxiety disorder, while $54 \%$ were diagnosed with more than one anxiety disorder (Brown et al., 2001). Among anxiety disorders, Generalized Anxiety Disorder (GAD) has the highest rate of comorbidity with other anxiety disorders, as well as other psychiatric conditions, which are associated with stress (Brown, O’Leary, \& Barlow, 2001).

A number of studies have investigated the comorbidity of GAD and Obsessive-Compulsive Disorder (OCD)(Gillan et al., 2017). Research reports that over
$30 \%$ of patients with OCD have a lifetime history of GAD (Crino \& Andrews, 1996). In addition, OCD and GAD share transdiagnostic cognitive vulnerabilities, symptomatology, and affective structures. Furthermore, they respond well to similar psychotherapeutic and psychopharmacological treatments (Comer, Kendall, Franklin, Hudson, \& Pimentel, 2004; Fineberg, et al., 2013; Goodwin, 2015; Torres et al., 2016). Freeston et al. suggested that obsessions particularly complicates the differential diagnosis between GAD and OCD. Furthermore, compulsive behaviors are present in GAD (Coleman, Pietrefesa, Holaway, Coles, \& Heimberg, 2011; Fineberg, et al., 2013). Considering the similarities between GAD and OCD, some scholars have called them "neighboring disorders" (Brown, Chorpita, $\&$ Barlow, 1998). The source of difficulty in the differential diagnosis is the commonality between obsessive thoughts and worry (Turner, Beidel, \& Stanley, 1992).

Historically, worry is the exclusive criterion in the diagnosis of GAD (Newman, Llera, Erickson, Przeworski, \& Castonguay, 2013). Worry is characterized by uncontrollable thoughts, fear, and images focusing on negative outcomes (Borkovec, 1994). Worry is also conceptualized as a cognitive component of anxiety that involves distorted information processing and attentional vigilance (Cisler $\&$ Koster, 2010). Findings on the exclusiveness of the relationship between worry and GAD are rather inconsistent. 
Several studies have identified worry in OCD (Fergus \& $\mathrm{Wu}, 2010$ ), as well as other psychological disorders (Gladstone et al., 2005). Evidence shows that worry, as a thought control strategy, is associated with Obsessive-Compulsive (OC) symptoms in non-clinical samples (McKay \& Greisberg, 2002). Some studies have suggested that worry is the exclusive thought control strategy in OCD as patients with OC symptoms report higher levels of worry compared with their non-clinical and non-OCD counterparts (Abramowitz, Whiteside, Kalsy, \& Tolin, 2003). On the contrary, in some other studies, patients with OCD do not report higher levels of worry compared with non-clinical samples, depressed samples, and other anxious groups (Belloch, Morillo, \& Garcia-Soriano, 2009). Closing the gap between controversial findings, transdiagnostic models of psychopathology (Barlow, et al., 2017; Borkovec, Alcaine, \& Behar, 2004; Dugas, Gagnon, Ladouceur, \& Freeston, 1998; Wells, 2005) are considered as the preferred conceptualization for explaining the shared components of different psychological disorders. In this regard, worry might play a common role in the development and or perpetuating different psychological disorders.

Various models have examined worry as a transdiagnostic process. Among such models, the Cognitive Avoidance Model (CAM) (Borkovec et al., 2004), Intolerance of Uncertainty Model (IUM) (Dugas et al., 1998), and Metacognitive Model (MCM) (Wells, 2005) are worth noting. Underlying cognitive and metacognitive factors as the shared components across these models are considered in developing unified intervention programs in various anxiety disorders. Broadly speaking, cognitive avoidance refers to one's tendency to suppress unwanted thoughts and avoiding disturbing thoughts or images (Dugas et al., 1998). Extremely worried individuals and patients with GAD actively engage in suppressing worry-related thoughts (Wells \& Papageorgiou, 1998). Patients with OCD also use suppression to control thoughts, which is paradoxically less perceived thought control (Tolin, Abramowitz, Brigidi, \& Foa, 2003). Furthermore, cognitive avoidance is associated with worry in clinical and non-clinical samples (Dugas, Marchand, \& Ladouceur, 2005). Compared with less worried individuals, extremely worried people use cognitive avoidance strategies more often (Gosselin et al., 2007). Cognitive avoidance plays a crucial role in developing GAD symptoms and these symptoms longitudinally enhance cognitive avoidance (Olatunji, Moretz, \& Zlomke, 2010). Similar to worry, Intolerance of Uncertainty (IU) is another common cognitive construct, and several clinical conditions, including DAG and OCD recognized it as a transdiagnostic factor (Holaway, Heimberg, \& Coles,
2006; Steketee, Frost, \& Cohen, 1998; Thibodeau, et al., 2015; Tolin et al., 2003).

IU refers to one's inability to accept the probability of an event in the future even when that event is unlikely (Dugas, Gosselin, \& Ladouceur, 2001) and has robust evolutionary roots (Brosschot, Verkuil, \& Thayer, 2016). Those who experience IU relate any uncertainty with negative outcomes that are stressful and disturbing (see Carleton, 2016 for a review); consequently, they avoid these situations. Dugas et al., suggested that IU serves as a regulation in the chain of worry, negative orientation toward problems, and cognitive avoidance. According to IU, ambiguity may automatically provoke worry toward catastrophic explanations and continuous distress (Dugas et al., 2005). In this situation, the people with higher $\mathrm{IU}$ might be prone to the process of worry.

IU may be involved in OCD and studies that are more recent indicate the similarity of the process in GAD and OCD (Tolin et al., 2003). Some scholars have suggested that patients with OCD seek certainty and experience considerable anxiety until certainty is somehow reassured (Holaway et al., 2006). In several studies, IU is associated with pathological worry, GAD symptoms, and OC symptoms (e.g. Tolin et al., 2003; Holaway et al., 2006). Both patients with GAD and OCD exhibit a higher rate of IU compared with the control group; however, the difference between patients with GAD and OCD is usually non-significant. On the other hand, some studies show that patients with OCD exhibit higher rates of IU compared with all other anxiety disorders, including GAD (Steketee et al., 1998). In addition, IU is the only construct (compared to responsibility, control, anxiety tolerance, and coping) that can predict OCD symptoms while controlling for mood and worry. Moreover, research suggests that severe worry and obsessions act as cognitive reactions to IU. This functional process may distinguish GAD from OCD and other anxiety disorders; however, it may not differentiate between GAD and OCD (see Thibodeau et al., 2015 for transdiagnostic features of IU and its components). IU does not belong to a certain disorder, but it is involved in both GAD and OCD.

Metacognitive beliefs are considered another cognitive construct that is associated with worry (Davey \& Meeten, 2016). There are positive and negative metacognitive beliefs about worry. Using worry as a coping strategy is related to positive metacognitive beliefs, while uncontrollability and danger of worry are considered negative metacognitive beliefs. With such beliefs, one may experience worry about worry, which is commonly known as meta-worry or 
type-2 worry (Pretorius, Walker, \& Esterhuyse, 2015). The worry in GAD with regard to negative beliefs about worry is distinct from ordinary worry (Wells, 2005). Multivariate analyses have shown that patients are significantly different from the control group regarding their beliefs on controllability and danger, cognitive sufficiency, and general negative beliefs. Barahmand like Wells suggested that metacognitive beliefs about worry were similar among people with worry. Therefore, metacognitive beliefs and strategies may draw a sharp distinction between patients and non-patients.

Wells identified metacognitive beliefs in the pathogenesis of OCD symptoms, consisting of thought-action fusion and metacognitive beliefs on the necessity of performing compulsive rituals. Furthermore, a positive relationship is found among the dimensions of metacognition, pathological worry, and OCD symptoms (Grøtte et al., 2015; Wells \& Papageorgiou, 1998). Positive and negative beliefs about worry may predict worry and obsessive thoughts. Another study indicated that obsessive symptoms are associated with the thoughts of danger and need for thought control (Borkovec \& Roemer, 1995). Belloch et al., (2007) also reported that patients with OCD have stronger desires for thought control and beliefs about the importance of thoughts compared to patients of other anxiety disorders and depression.

Based on the above-mentioned literature, the present study aimed at examining worry, cognitive avoidance, IU, and metacognitive beliefs, as transdiagnostic processes, in GAD and OCD. Investigating common cognitive aspects of these disorders is particularly important as they can be used in transdiagnostic approaches that design the treatment plans based on unifying common cognitive processes among psychological disorders. Unified therapies are more convenient and economic (Barlow et al., 2017; Norton \& Paulus, 2017; Talkovsky and Norton, 2016), and encounter higher rates of acceptance with a concentration on common processes (Newby et al., 2016). As argued by Gillan et al., a transdiagnostic framework can be a rational approach to advance basic science research in psychopathology. After exclusion of OCD from the anxiety disorders category in the Diagnostic and Statistical Manual of Mental Disorders, Fifth Edition (DSM-5; APA, 2013), we need to identify the possible transdiagnostic factors or endophenotypes of GAD and OCD. Moreover, possible differences between patients with GAD and OCD in terms of the research variables could call for specific interventional techniques. In this regard, the role of transdiagnostic underlying factors of OCD and GAD is also important and may serve to improve understanding of their diagnoses and treatments. These can be considered an additional contribution of the present to the existing literature on unified transdiagnostic protocols for the treatment of anxiety disorders. The present study sought to answer the following questions:

1. Are worry, cognitive avoidance, IU, and metacognitive beliefs transdiagnostic factors in GAD and OCD?; 2. Are worry, cognitive avoidance, IU, and metacognitive beliefs more severe in patients with comorbid GAD and OCD compared with the patients with GAD or OCD?; 3. Are metacognitive beliefs different in patients with GAD, OCD, and comorbid GAD and OCD? and 4. Are cognitive avoidance strategies different in patients with GAD, OCD, and comorbid GAD and OCD?

\section{Methods}

The present research is a descriptive study. The study population included psychiatric patients. Using purposeful sampling method (a non-probability sampling method that is widely used to identify and select information-rich or criterion-satisfying cases related to the phenomenon of interest; Yin, 2009), 3 groups of outpatients (patients with GAD, $\mathrm{OCD}$, and comorbid GAD and $\mathrm{OCD}$ ) were recruited from two psychiatric hospitals in two major cities in Iran. The participants were asked to take part in a "study on characteristics of people having GAD and or OCD" via their psychiatrist secretary.

The inclusion criteria included patients 18 years or older, with GAD, OCD, or their comorbidity by a certified psychiatrist (made by diagnostic criteria of the Diagnostic and Statistical Manual of Mental Disorders, Fifth Edition; APA, 2013), without psychotic disorder, no intervention except the ongoing psychiatric treatment, and the consent to take part in the study.

The final sample included 66 patients with GAD, 74 patients with OCD, and 60 patients with both GAD and OCD. In these groups, 52, 51, and 49 participants were women, respectively. Moreover, the mean age of the groups was 33.2 $(\mathrm{SD}=10.24), 33.7(\mathrm{SD}=10.14)$, and $34.6(\mathrm{SD}=9.55)$ years, respectively. The age was not significantly different between the groups $(\mathrm{P}>0.05)$. The Department of Psychology of the University of Tehran approved the protocol. All participants signed informed consent beforehand. After explaining the subject and the aim of the research to the participants, they completed the questionnaires. Furthermore, assurance was given on the confidentiality of results.

\section{Penn State Worry Questionnaire (PSWQ)}

Penn State Worry Questionnaire (PSWQ) is a 16-item self-report scale for the assessment of pathological worry directed at the excessiveness, uncontrollability, and 
duration of worry. A sample item is "Once I start worrying, I can't stop.” Items are scored with a 5-point scale ranging from 1 (completely disagree) to 5 (completely agree). The PSWQ has demonstrated high reliability, as well as high temporal stability and substantial validity in the assessment of trait worry (Meyer, Miller, Metzger, \& Borkovec, 1990). In the present study, the internal consistency coefficient was excellent (Cronbach's $\alpha=0.88$ ).

\section{Cognitive Avoidance Questionnaire (CAQ)}

The Cognitive Avoidance Questionnaire (CAQ) (Gosselin et al., 2002) consists of 25 questions assessing 5 cognitive avoidance strategies (thought substitution, the transformation of images into verbal thoughts, distraction, avoidance of stimuli that trigger unpleasant thoughts, and thought suppression). All items are scored based on a 5-point Likerttype scale. The assessment of the CAQ's psychometric properties supports its 5-factor structure. Research suggests that the overall scale and its subscales have satisfactory reliability and validity (Gosselin et al., 2002). In the present study, we used the total score (Cronbach's $\alpha=0.92$ ).

\section{Intolerance of Uncertainty Scale}

The Intolerance of Uncertainty Scale-12 (IUS) is a 12item short-form of the original 27-item IUS (Freeston et al., 1994) that measures reactions to uncertainty, ambiguous situations, and the future. Items are scored based on a 5-point Likert scale ranging from 1 (not at all characteristic of me) to 5 (entirely characteristic of me). This instrument assesses trait IU, rather than situationspecific IU. The IUS-12 is psychometrically comparable to the original 27-item IUS (Khawaja \& Yu, 2010). The IUS-12 and its two subscales have demonstrated substantial transdiagnostic psychometric support (Jacoby, Fabricant, Leonard, Riemann, \& Abramowitz, 2013).
The internal consistency of the whole scale was found high in this study (Cronbach's $\alpha=0.89$ ).

\section{Metacognitive Beliefs Questionnaire}

The Metacognitive Beliefs Questionnaire (MCQ-30) (Wells \& Cartwright-Hatton, 2004) is a self-report measure of metacognitive beliefs. It consists of 30 items for assessing individual differences in metacognitive beliefs. The items are scored based on a 4-point Likert scale ranging from 1 (I do not agree) to 4 (I totally agree). Therefore, the total score may vary between 30 and 120 . These 30 items are grouped within 5 subscales of positive beliefs about worry, negative beliefs about worry concerning uncontrollability and danger, cognitive confidence, the need for control, and cognitive self-consciousness, which is the tendency to be aware of one's thoughts. MCQ-30 has shown good reliability (Wells \& Cartwright-Hatton, 2004). In the present study, the Cronbach's alpha values for the subscales ranged from 0.71 (need of control) to 0.86 (positive beliefs about worry).

\section{Results}

Table 1 presents the mean and SD of all variables (worry, cognitive avoidance, IU, and metacognitive beliefs). Four distinct one-way ANOVA tests showed that worry, cognitive avoidance, IU, and metacognitive beliefs were significantly different across the groups (Table 1). The magnitude of all relationships was substantial, indicating significant differences in the mean scores of the measures across groups.

Table 2 summarizes Scheffe post hoc test for the comparison of the groups in all variables. The mean scores of worry, cognitive avoidance, IU, and metacognitive beliefs are not significantly different $(\mathrm{P}>0.05)$ between patients with GAD and OCD. Yet, all variables are con-

Table 1. Descriptive statistics and comparison of the variables

\begin{tabular}{|c|c|c|c|c|c|c|c|c|}
\hline \multirow{2}{*}{$\begin{array}{c}\text { variables } \\
\text { worry }\end{array}$} & \multicolumn{2}{|c|}{ Patients with GAD } & \multicolumn{2}{|c|}{ Patients with OCD } & \multicolumn{2}{|c|}{ Comorbidity Group } & \multirow{2}{*}{$\begin{array}{c}\mathbf{F} \\
46.34^{*}\end{array}$} & \multirow{2}{*}{$\begin{array}{l}\boldsymbol{\eta} \mathbf{2}_{\mathrm{p}} \\
0.33\end{array}$} \\
\hline & 53.04 & 13.11 & 51.14 & 14.66 & 69.75 & 14.82 & & \\
\hline $\mathrm{CA}$ & 68.77 & 18.31 & 65.67 & 18.80 & 81.23 & 17.39 & $13.06^{*}$ & 0.12 \\
\hline IU & 38.48 & 13.98 & 37.68 & 13.45 & 52.38 & 11.58 & $25.12^{*}$ & 0.20 \\
\hline MCB & 81.19 & 12.83 & 75.85 & 16.57 & 92.36 & 11.08 & $23.90 *$ & 0.19 \\
\hline
\end{tabular}

CA: Cognitive Avoidance; IU: Intolerance of Uncertainty; MCB: Metacognitive Beliefs; GAD: Generalized Anxiety Disorder; OCD: Obsessive-Compulsive Disorder

${ }^{*} \mathrm{P}<0.01$ (P-values are Bonferroni-corrected) 
Table 2. The mean difference between the variables

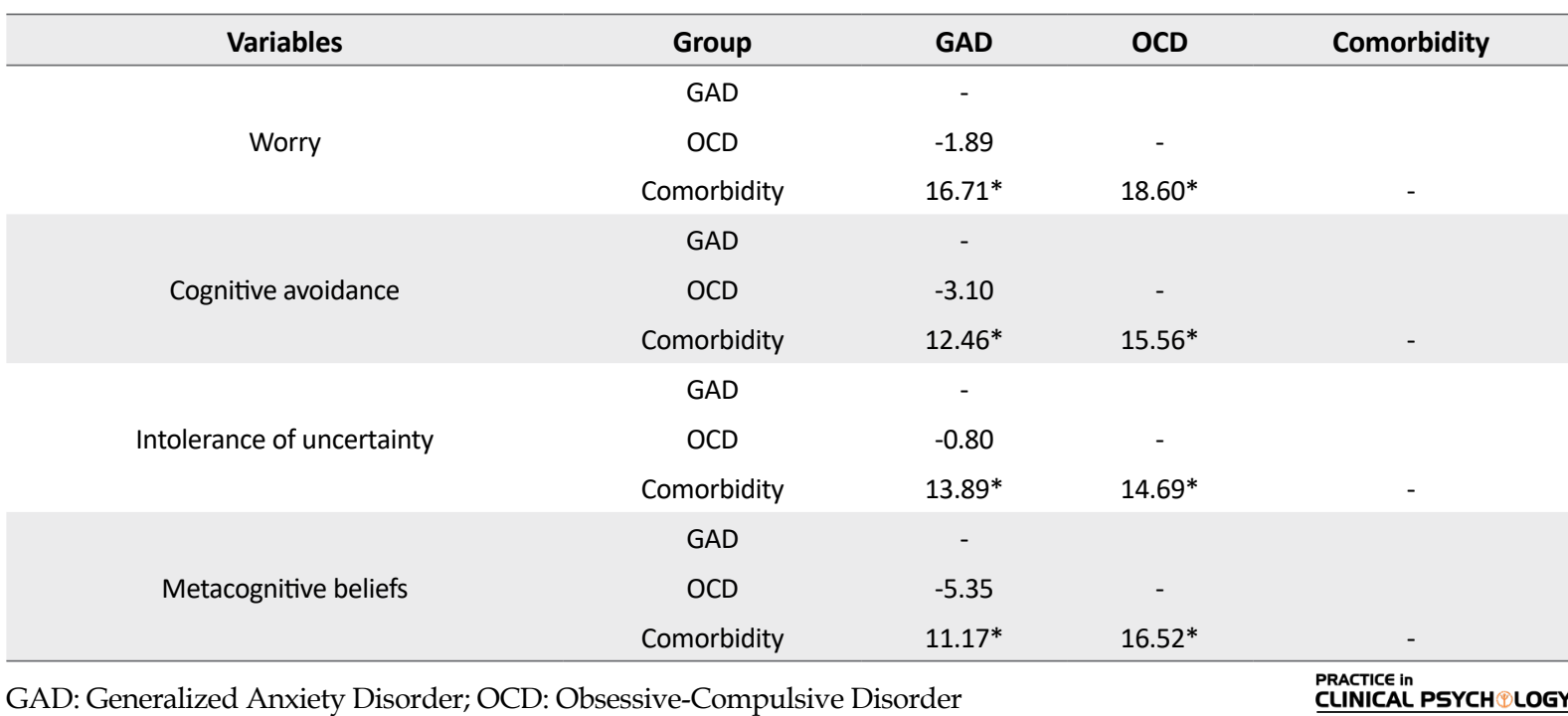

${ }^{*} \mathrm{P}<0.05$

siderably higher $(\mathrm{P}<0.05)$ in patients with comorbid GAD and OCD. Patients with comorbid GAD and OCD scored higher on transdiagnostic constructs utilized in this study (Table 2).

We also conducted 5 distinct MANOVA tests to compare scores of the subscales of the metacognitive beliefs. Negative beliefs about worry was significantly different $(\mathrm{F}=6.48$, $\left.\mathrm{P}<0.05, \eta 2_{\mathrm{p}}=0.06\right)$. Other subscales were not significantly different $(\mathrm{P}>0.05)$. The mean score of the negative beliefs was significantly higher in the comorbidity group $(\mathrm{P}<0.05)$.

We conducted 5 MANOVA tests to examine betweengroup differences in scores of 5 subscales of cognitive avoidance. The transformation of images into verbal thoughts was significantly different $(\mathrm{F}=4.17, \mathrm{P}<0.05$, $\left.\eta 2_{\mathrm{p}}=0.04\right)$. The mean score of the negative beliefs was significantly higher among patients with GAD $(\mathrm{P}<0.05)$. The difference between the comorbidity group and other groups was statistically non-significant $(\mathrm{P}>0.05)$.

\section{Discussion}

The present study aimed at examining transdiagnostic factors in GAD and OCD. Analyses suggested that patients with GAD and OCD did not significantly differ in worry. Yet, comorbidity of GAD and OCD was strongly associated with higher levels of worry. The magnitude of this difference was considerable. This finding is consistent with studies conducted by Fergus and Wu and Barahmand. Patients with OCD use worry as their primary strategy of thought control. Based on the previous research on the cooccurrence of chronic worry and obsessive thoughts (Wells
\& Papageorgiou, 1998), worry may not serve as a distinguishing factor in the diagnosis of GAD and OCD. In other words, both patients with GAD and OCD experience high levels of worry. Therefore, worry maybe a transdiagnostic underlying factor of the symptoms of GAD and OCD, so it should be taken into account in the treatment of both disorders, particularly using unified transdiagnostic protocols.

Findings on cognitive avoidance indicated that patients with GAD and OCD did not significantly differ; however, the comorbidity of GAD and OCD was associated with higher levels of cognitive avoidance. To our knowledge, the present study is the first research that compares cognitive avoidance between patients with GAD and OCD. Findings concerning different cognitive avoidance strategies revealed that patients with GAD scored higher on the transformation of images into thoughts compared with the patients with OCD. This finding is consistent with previous studies (Borkovec et al., 2004), reporting higher verbal activity as an emotion-controlling strategy in patients with GAD. Although cognitive avoidance may be considered a transdiagnostic factor in GAD and OCD, specific transformation-related techniques should be implemented in therapeutic models for patients with GAD.

Findings regarding metacognitive beliefs indicated that the difference between patients with GAD and OCD was non-significant in comparison with the total score of metacognitive beliefs and its subscales. Additionally, the patients with comorbid GAD and OCD scored higher in total metacognitive beliefs, as well as their negative belief subscale. These findings are in line with previous studies (Barahmand, 2009), suggesting that patients with 
GAD and OCD score higher in metacognitive beliefs. As a result, metacognitive beliefs may be considered another transdiagnostic factor in the diagnosis and treatment of GAD and OCD.

Findings on the IU suggested that patients with GAD and OCD did not significantly differ in IU. Yet, patients with both GAD and OCD reported higher IU. These findings suggest that patients who met the criteria for both disorders had higher intolerance toward uncertainty. Holaway et al. indicated that higher levels of IU are associated with more severe psychopathological symptoms. Moreover, IU has a unique relationship with symptoms of GAD and OCD and that their comorbidity may increase patients' scores of selfreported IU.

Base on the present study, worry, cognitive avoidance, IU, and metacognitive beliefs may be considered transdiagnostic factors in the diagnosis and treatment of GAD and OCD. Future research may examine the association between these constructs and anxiety symptoms, as well as obsessive-compulsive dimensions (Fineberg et al., 2013; Gillan et al., 2017; Rufer, Fricke, Moritz, Kloss, \& Hand, 2006; Torres et al., 2016). The findings of the present study also highlighted the notion that comorbidity of GAD and OCD may strengthen the magnitude of maladaptive cognitive components of these disorders. If maladaptive cognitive components of GAD and OCD are amplified by comorbidity, both must be considered a diagnostic criterion and a measure of treatment outcome.

One practical implication of the present study is the utilization of these common transdiagnostic factors in unified therapy (a mixed protocol comprising several fundamental therapeutic components), which is both economical and popular among patients (Barlow, Allen, \& Choate, 2004; Barlow et al., 2017). Since the symptoms of anxiety disorders are inter-related and shared features in nature, identifying transdiagnostic factors may help therapists to overcome such challenges in diagnosis and course of treatment. Transdiagnostic approaches and unified therapies are associated with high patient satisfaction, positive expectations, and integration of the treatment process (McEvoy, Nathan, \& Norton, 2009). Furthermore, identifying common cognitive aspects of anxiety disorders and their role in psychopathology may improve the insight into the design and implementation of prevention programs (Dozois, Seeds, \& Collins, 2009).

Some limitations of the present study are worth noting. Firstly, the sampling method was based on voluntary participation and non-probability sampling from limited settings. Therefore, generalizing the findings should be made with caution. Secondly, the methodology of the present study was correlational. Thus, directional attributions should be treated with caution. Thirdly, although a certified psychiatrist diagnosed the GAD and OCD, caution must be taken because no structured diagnostic instrument was used. Fourthly, other comorbid disorders that frequently co-occur with GAD and OCD were not assessed. These disorders could have a confounding influence on the results of the study. Fifthly, the severity of the disorders was not assessed. For future research, it is recommended to use probability sampling methods, as well as longitudinal designs for the evaluation of these transdiagnostic processes in GAD and OCD. Moreover, future studies may include other predictors of worry (negative orientation toward problems and attentional bias) as potential transdiagnostic factors of GAD and OCD.

In sum, the present study highlighted the significance of the transdiagnostic roles of worry, cognitive avoidance, IU, and metacognitive beliefs in GAD and OCD as prevalent psychological disorders. Findings may contribute to the existing literature on unified transdiagnostic protocols for the treatment of anxiety disorders, particularly GAD and OCD. However, specific interventional techniques such as transformation-related techniques might maximize outcomes for patients with GAD rather than patients with OCD. Future research should address this particular interventional technique for patients with GAD. Informed consent was obtained from all participants of the study.

\section{Ethical Considerations}

\section{Compliance with ethical guidelines}

All procedures performed in this study are according to the ethical standards of the responsible committee on human experimentation and the 1964 Declaration of Helsinki.

\section{Funding}

The study received no financial support.

\section{Authors' contributions}

Conceptualization and design: Author A; data analysis and initial draft preparation: Author B; Resources and data analysis: Authors $\mathrm{C}$; final draft preparation: All authors

\section{Conflict of interest}

The authors declared no conflict of interest. 


\section{References}

Abramowitz, J. S., Whiteside, S., Kalsy, S. A., \& Tolin, D. F. (2003). Thought control strategies in obsessive-compulsive disorder: A replication and extension. Behaviour Research and Therapy, 41(5), 529-40. [DOI:10.1016/S0005-7967(02)00026-8]

Barahmand, U. (2009). Meta-cognitive profiles in anxiety disorders. Psychiatry Research, 169(3), 240-3. [DOI:10.1016/j.psychres.2008.06.029] [PMID]

Barlow, D. H., Allen, L. B., \& Choate, M. L. (2004). Toward a unified treatment for emotional disorders. Behavior Therapy, 35(2), 205-30. [DOI:10.1016/S0005-7894(04)80036-4]

Barlow, D. H., Farchione, T. J., Bullise, J. R., Gallagher, M. W., Murray-Latin, H., Sauer-Zavala, S., et al. (2017). The unified protocol for transdiagnostic treatment of emotional disorders compared with diagnosis-specific protocols for anxiety disorders: A randomized clinical trial. JAMA Psychiatry, 74(9), 875-84. [DOI:10.1001/jamapsychiatry.2017.2164] [PMID] [PMCID]

Belloch, A., Morillo, C., \& Garcia-Soriano, G. (2007). Are the dysfunctional beliefs that predict worry difference from those that predict obsessions? Clinical Psychology $\mathcal{E}$ Psychotherapy, 14(6), 438-48. [DOI:10.1002/cpp.551]

Belloch, A., Morillo, C., \& Garcia-Soriano, G. (2009). Strategies to control unwanted intrusive thoughts: Which are relevant and specific to obsessive-compulsive disorder? Cognitive Therapy and Research, 33, 75-89. [DOI:10.1007/s10608-007-9141-2]

Borkovec, T. D. (1994). The nature, functions, and origins of worry. In G. C. Davey, \& F. Tallis (Eds.), Worrying: Perspectives on theory, assessment, and treatment (pp. 5-33). Chichester: John Wiley \& Sons.

Borkovec, T. D., \& Roemer, L. (1995). Perceived functions of worry among generalized anxiety disorder subjects: Distraction from more emotionally distressing topics? Journal of Behavior Therapy and Experimental Psychiatry, 26(1), 25-30. [DOI:10.1016/0005-7916(94)00064-S]

Borkovec, T. D., Alcaine, O. M., \& Behar, E. (2004). Avoidance theory of worry and generalized anxiety disorder. In R. G. Heimberg, C. L. Turk, \& D. S. Mennin (Eds.), Generalized anxiety disorder: Advances in research and practice (pp. 77-108). New York: Guilford Press.

Brosschot, J. F., Verkuil, B., \& Thayer, J. F. (2016). The default response to uncertainty and the importance of perceived safety in anxiety and stress: An evolution-theoretical perspective. Journal of Anxiety Disorders, 41, 22-34. [DOI:10.1016/j. janxdis.2016.04.012] [PMID]

Brown, T. A., Campbell, L. A., Lehman, C. L., Grisham, J. R., \& Mancill, R. B. (2001). Current and lifetime comorbidity of the DSM-IV anxiety and mood disorders in a large clinical sample. Journal of Abnormal Psychology, 110(4), 585-99. [DOI:10.1037/0021-843X.110.4.585] [PMID]

Brown, T. A., Chorpita, B. F., \& Barlow, D. H. (1998). Structura relationships among dimensions of the DSM-IV anxiety and mood disorders and dimensions of negative affect, positive affect and autonomic arousal. Journal of Abnormal Psychology, 107(2), 179-92. [DOI:10.1037/0021-843X.107.2.179] [PMID]

Brown, T. A., O'Leary, T. A., \& Barlow, D. H. (2001). Generalized anxiety disorder. In D. H. Barlow (Ed.), Clinical handbook of psychological disorders: A step by step treatment manual (pp. 154-208). $3^{\text {rd }}$ Ed. New York, NY: Guilford Press.

Carleton, R. N. (2016). Into the unknown: A review and synthesis of contemporary models involving uncertainty. Journal of Anxiety Disorders, 39, 30-43. [DOI:10.1016/j. janxdis.2016.02.007] [PMID]

Cisler, J. M., \& Koster, E. H. (2010). Mechanisms of attentiona biases towards threat in anxiety disorders: An integrative review. Clinical Psychology Review, 30(2), 203-16. [DOI:10.1016/j. cpr.2009.11.003] [PMID] [PMCID]

Coleman, S. L., Pieterefesa, A. S., Holaway, R. M., Coles, M. E., \& Heimberg, R. G. (2011). Content and correlates of checking related to symptoms of obsessive compulsive disorder and generalized anxiety disorder. Journal of Anxiety Disorders, 25(2), 293-301. [DOI:10.1016/j.janxdis.2010.09.014] [PMID]

Comer, J. S., Kendall, P. C., Franklin, M. E., Hudson, J. L., \& Pimentel, S. S. (2004). Obsessing/worrying about the overlap between obsessive-compulsive disorder and generalized anxiety disorder in youth. Clinical Psychology Review, 24(6) 663-83. [DOI:10.1016/j.cpr.2004.04.004] [PMID]

Crino, R. C., \& Andrews, G. (1996). Obsessive-compulsive disorder and axis I comorbidity. Journal of Anxiety Disorders, 10(1), 37-46. [DOI:10.1016/0887-6185(95)00033-X]

Davey, G. C., \& Meeten, F. (2016). The perseverative worry bout: A review of cognitive, affective and motivational factors that contribute to worry perseveration. Biological Psychol ogy, 121(Pt B), 233-43. [DOI:10.1016/j.biopsycho.2016.04.003] [PMID]

Dozois, D. J. A., Seeds P. M., \& Collins, K. A. (2009). Transdiagnostic approaches to the prevention of depression and anxiety. Journal of Cognitive Psychotherapy: An International Quarterly, 23(1), 44-59. [DOI:10.1891/0889-8391.23.1.44]

Dugas, M. J., Gagnon, F., Ladouceur, R., \& Freeston, M. H (1998). Generalized anxiety disorder: A preliminary test of a conceptual model. Behavior Research and Therapy, 36(2), 215-26. [DOI:10.1016/S0005-7967(97)00070-3]

Dugas, M. J., Gosselin, P., \& Ladouceur, R. (2001). Intolerance of uncertainty and worry: Investigating specificity in a nonclinical sample. Cognitive Therapy and Research, 25(5), 551-8. [DOI:10.1023/A:1005553414688]

Dugas, M. J., Marchand, A., \& Ladouceur, R. (2005). Further validation of a cognitive-behavioral model of generalized anxiety disorder: Diagnostic and symptom specificity. Journal of Anxiety Disorders, 19(3), 329-43. [DOI:10.1016/ j.janxdis.2004.02.002] [PMID]

Fergus, T. A., \& Wu, K. D. (2010). Do symptoms of generalized anxiety and obsessive-compulsive disorder share cognitive processes? Cognitive Therapy and Research, 34(2), 168-76. [DOI:10.1007/s10608-009-9239-9]

Fineberg, N. A., Hengartner, M. P., Bergbaum, C., Gale, T. Rössler, W., Angst, J. (2013). Lifetime comorbidity of obsessive-compulsive disorder and sub-threshold obsessivecompulsive symptomatology in the community: Impact, prevalence, socio-demographic and clinical characteristics. International Journal of Psychiatry and Clinical Practice, 17(3), 188-96. [DOI:10.3109/13651501.2013.777745] [PMID]

Freeston, M. H., Rhéaume, J., Letarte, H., Dugas, M. J., \& Ladouceur, R. (1994). Why do people worry? Personality 
and Individual Differences, 17(6), 791-802. [DOI:10.1016/01918869(94)90048-5]

Gillan, C. M., Fineberg, N. A., \& Robbins, T. W. (2017). A trans-diagnostic perspective on obsessive-compulsive disorder. Psychological Medicine, 47(9), 1528-48. [DOI:10.1017/ S0033291716002786] [PMID] [PMCID]

Gladstone, G. L., Parker, G. B., Mitchell, P. B., Malhi, G. S., Wilhelm, K. A. \& Austin, M. P. (2005). A Brief Measure of Worry Severity (BMWS): Personality and clinical correlates of severe worriers. Journal of Anxiety Disorders, 19(8), 877-92. [DOI:10.1016/j.janxdis.2004.11.003] [PMID]

Goodwin, G. M. (2015). The overlap between anxiety, depression, and obsessive-compulsive disorder. Dialogues in Clinical Neuroscience, 17(3), 249-60. [PMID] [PMCID]

Gosselin, P., Langlois, F., Freeston, M. H., Ladouceur, R., Dugas, M. J., \& Pelletier, O. (2002). The Cognitive Avoidance Questionnaire (CAQ): Development and validation among adult and adolescent samples. Journal de Thérapie Comportementale et Cognitive, 12(1), 24-37.

Gosselin, P., Langlois, F., Freeston, M. H., Ladouceur, R., Laberge, M., \& Lemay, D. (2007). Cognitive variables related to worry among adolescents: Avoidance strategies and faulty beliefs about worry. Behaviour Research and Therapy, 45(2), 22533. [DOI:10.1016/j.brat.2006.03.001] [PMID]

Grøtte, T., Solem, S., Vogel, P. A., Güzey, I. C., Hansen, B., \& Myers, S. G. (2015). Metacognition, responsibility, and perfectionism in obsessive-compulsive disorder. Cognitive Therapy and Research, 39(1), 41-50. [DOI:10.1007/s10608-014-9635-7]

Harvey, A., Watkins, E., Mansell, W., \& Shafran, R. (2004). Cognitive behavioral processes across disorders: A transdiagnostic approach to research and treatment. Oxford, UK: Oxford University Press. [DOI:10.1093/med:psych/9780198528883.001.0001]

Holaway, R. M., Heimberg, R. G., \& Coles, M. E. (2006). A comparison of intolerance of uncertainty in analogue obsessive-compulsive disorder and generalized anxiety disorder. Journal of Anxiety Disorders, 20(2), 158-74. [DOI:10.1016/j. janxdis.2005.01.002] [PMID]

Jacoby, R. J., Fabricant, L. E., Leonard, R. C., Riemann, B. C., \& Abramowitz, J. S. (2013). Just to be certain: Confirming the factor structure of the intolerance of uncertainty scale in patients with obsessive-compulsive disorder. Journal of Anxiety Disorders, 27(5), 535-42. [DOI:10.1016/j.janxdis.2013.07.008] [PMID]

Khawaja, N. G., \& Yu, L. N. H. (2010). A comparison of the 27item and 12-item intolerance of uncertainty scales. Clinical Psychologist, 14(3), 97-106. [DOI:10.1080/13284207.2010.502542]

Mansell, W., Harvey, A., Watkins, E. R., \& Shafran, R. (2008). Cognitive behavioral processes across psychological disorders: A review of the utility and validity of the transdiagnostic approach. International Journal of Cognitive Therapy, 1(3), 181-91. [DOI:10.1521/ijct.2008.1.3.181]

McEvoy, P. M., Nathan, P., \& Norton, P. J. (2009). Efficacy of transdiagnostic treatments: A review of published outcome studies and future research directions. Journal of Cognitive Psychotherapy: An International Quarterly, 23(1), 20-33. [DOI:10.1891/0889-8391.23.1.20]
McKay, D., \& Greisberg, S. (2002). Specificity of measures of thought control. The Journal of Psychology, 136(2), 149-60. [DOI:10.1080/00223980209604146] [PMID]

McLaughlin, K. A., Nolen-Hoeksema, S. (2011). Rumination as a transdiagnostic factor in depression and anxiety. Behavior Research and Therapy, 49(3), 186-93. [DOI:10.1016/j. brat.2010.12.006] [PMID] [PMCID]

Meyer, T. J., Miller, M. L., Metzger, R. L., \& Borkovec, T. D. (1990). Development and validation of the Penn State Worry Questionnaire. Behavior Research and Therapy, 28(6), 487-95. [DOI:10.1016/0005-7967(90)90135-6]

Newby, J. M., Twomey, C., Li, S. S. Y., \& Andrews, G. (2016) Transdiagnostic computerised cognitive behavioral therapy for depression and anxiety: A systematic review and meta-analysis. Journal of Affective Disorders, 199, 30-41. [DOI:10.1016/j.jad.2016.03.018] [PMID]

Newman, M. G., Llera, S. J., Erickson, T. M., Przeworski, A., \& Castonguay, L. G. (2013). Worry and generalized anxiety disorder: A review and theoretical synthesis of evidence on nature, etiology, mechanisms, and treatment. Annual Review of Clinical Psychology, 9, 275-97. [DOI:10.1146/annurevclinpsy-050212-185544] [PMID] [PMCID]

Norton, P. J., \& Paulus, D. J. (2017). Transdiagnostic models of anxiety disorder: Theoretical and empirical underpinnings. Clinical Psychology Review, 56, 122-137. [Doi: 10.1016/j. cpr.2017.03.004].

Olatunji, B. O., Moretz, M. W., \& Zlomke, K. R. (2010). Linking cognitive avoidance and GAD symptoms: The mediating role of fear of emotion. Behavior Research and Therapy, 48(5), 435-41. [DOI:10.1016/j.brat.2009.11.014] [PMID]

Pretorius, C., Walker, S. P., \& Esterhuyse, K. G. (2015). The applicability of the metacognitive model of worry and generalized anxiety disorder in a non-clinical multi-ethnic sample of university students. South African Journal of Psychology, 45(2), 234-48. [DOI:10.1177/0081246314567890]

Rufer, M., Fricke, S., Moritz, S., Kloss, M., \& Hand, I. (2006). Symptom dimensions in obsessive-compulsive disorder: Prediction of cognitive-behavior therapy outcomes. Acta Psychiatrica Scandinavica, 113(5), 440-6. [DOI:10.1111/j.16000447.2005.00682.x] [PMID]

Steketee, G., Frost, R. O., \& Cohen, I. (1998). Beliefs in obsessivecompulsive disorder. Journal of Anxiety Disorders, 12(6), 52537. [DOI:10.1016/S0887-6185(98)00030-9]

Talkovsky, A. M., \& Norton, P. J. (2016). Intolerance of uncertainty and transdiagnostic group cognitive behavioral therapy for anxiety. Journal of Anxiety Disorders, 41, 108-14. [DOI:10.1016/j.janxdis.2016.05.002] [PMID]

Thibodeau, M. A., Carleton, R. N., McEvoy, P. M., Zvolensky, M. J., Brandt, C. P., \& Boelen, P. A., et al. (2015). Developing scales measuring disorder-specific intolerance of uncertainty (DSIU): A new perspective on transdiagnostic. Journal of Anxiety Disorders, 31, 49-57. [DOI:10.1016/j.janxdis.2015.01.006] [PMID]

Tolin, D. F., Abramowitz, J. S., Brigidi, B. D., \& Foa, E. B. (2003). Intolerance of uncertainty in obsessive-compulsive disorder Journal of Anxiety Disorders, 17(2), 233-42. [DOI:10.1016/ S0887-6185(02)00182-2] 
Torres, A. R., Fontenelle, L. F., Shavitt, R. G., Ferrão, Y. A., do Rosário, M. C., \& Storch, E. A., et al. (2016). Comorbidity variation in patients with obsessive-compulsive disorder according to symptom dimensions: Results from a large multicentre clinical sample. Journal of Affective Disorders, 190, 508-16. [DOI:10.1016/j.jad.2015.10.051] [PMID]

Turner, S. M., Beidel, D. C., \& Stanley, M. A. (1992). Are obsessional thoughts and worry different cognitive phenomena? Clinical Psychology Review, 12(2), 257-70. [DOI:10.1016/02727358(92)90117-Q

Wells, A. (2005). Worry, intrusive thoughts, and generalized anxiety disorder: The metacognitive theory and treatment. In D. A. Clark (Ed.), Intrusive thoughts in clinical disorders: Theory, research, and treatment (pp. 119-44). New York: Guilford Press.

Wells, A., \& Cartwright-Hatton, S. (2004). A short form of the metacognitions questionnaire: Properties of the MCQ-30. Behavior Research and Therapy, 42(4), 385-96. [DOI:10.1016/ S0005-7967(03)00147-5]

Wells, A., \& Papageorgiou, C. (1998). Relationships between worry, obsessive-compulsive symptoms and meta-cognitive beliefs. Behavior Research and Therapy, 36(9), 899-913. [DOI:10.1016/S0005-7967(98)00070-9]

Yin, R. (2009). Case study research: Design and methods. Thousand Oaks, CA: Sage. 